\title{
Inhibitory Effect of Japanese Traditional Kampo Formula Frequently Prescribed in Gynecological Clinics on CYP3A4
}

\author{
Hao Ni, ${ }^{1}$ Takashi Matsumoto, ${ }^{2}$ Junko Watanabe, ${ }^{2}$ and Toshiaki Makino ${ }^{1}{ }^{1}$ \\ ${ }^{1}$ Department of Pharmacognosy, Graduate School of Pharmaceutical Sciences, Nagoya City University, 3-1 Tanabe-dori, Mizuho-ku, \\ Nagoya 467-8603, Japan \\ ${ }^{2}$ Tsumura Kampo Research Laboratories, Kampo Research \& Development Division, Tsumura \& Co., 3586 Yoshiwara, Ami-machi, \\ Inashiki-gun, Ibaraki 300-1192, Japan
}

Correspondence should be addressed to Toshiaki Makino; makino@phar.nagoya-cu.ac.jp

Received 6 June 2018; Revised 13 September 2018; Accepted 23 September 2018; Published 1 October 2018

Academic Editor: Srinivas Nammi

Copyright (C) 2018 Hao Ni et al. This is an open access article distributed under the Creative Commons Attribution License, which permits unrestricted use, distribution, and reproduction in any medium, provided the original work is properly cited.

Recently, the use of herbal medicines has become popular, and information on drug interactions between herbal medicines and chemical drugs is needed in clinics. In Japan, the number of patients taking Japanese traditional Kampo medicines has been increasing, and the proper drug information about herb-drug interaction is highly demanded. The most established herb-drug interaction is the case of grapefruit juice (GFJ) via the inhibition on CYP3A4 expressed in the small intestine. In the present study, we compared the inhibitory titer on CYP3A4 between the target Kampo products and GFJ used as positive control. We evaluated the inhibitory effects of GFJ and three extracts of Kampo formulas frequently used in gynecological clinics on CYP3A4 in vitro and calculated the related titer of one-time dosage of Kampo formulas to GFJ in order to predict its effect on clinics. Although the extracts of these three Kampo formulas and the most of crude drug components in the formulas exhibited the inhibitory effects on CYP3A4 in some levels, the possibilities of tokishakuyakusan and keishibukuryogan to cause drug interaction can be quite low; however, it is possible that the excessive dosage of kamishoyosan may cause drug interaction with the substrate of CYP3A4 in clinics.

\section{Introduction}

As the integrated medicine using both modern Western medicine and traditional medicines is getting popular, the possibilities of the interaction between them have been described [1-4]. Especially in Japan, the number of patients taking Japanese traditional Kampo medicines has been increasing, and drug information about modern medicine and Kampo medicine is highly required. The most famous drug interaction between herbal and chemical drugs is the case of grapefruit juice (GFJ) [5]. Furanocoumarins such as 6,7'-dihydroxybergamottin (DHBG) contained in GFJ inhibit CYP3A4 expressed in the small intestine, which results in the augmentation of the blood concentration of some drugs and their activities in the body [6]. Some Kampo medicines containing crude drugs derived from Umbelliferae plants containing furanocoumarins have a potent inhibitory effect on CYP3A4 in vitro; however, these medicines did not cause drug interaction in rats $[7,8]$. In previous studies, excessive dosage of Kampo medicines may inhibit or induce CYP3A4 in animal experiments or in in vitro study [912]; it is predicted that their effects are too week not to cause drug interaction in clinic [13]. Since the results of CYP inhibitions in in vitro experiments tended to exhibit excessive high potency, only a few show interactions large enough to be clinically important [14]. Indeed, the clinical studies to evaluate drug interaction between Kampo and modern medicines must exhibit the best drug information; however, it is very limited to conduct clinical studies by cost and ethical problems.

In order to provide correct drug information about herbdrug interaction via CYP3A4 inhibition to physicians and pharmacists, we consider the comparison of the inhibitory titer of CYP3A4 between the target herbal products and GFJ, since GFJ is the only natural substance to inhibit CYP3A4 in clinical studies and used as positive control 
in many clinical studies [14]. Kampo medicines have been used as the ethical or OTC formulations in Japan, and the ingredients, methods of preparation, and dosages are approved by Japanese Medicine Regulatory Agency [15]. And in Japan, the most users of traditional Kampo medicine are the physicians belonging to gynecologics [16]. In this study, we evaluated the inhibitory effects of GFJ and three Kampo medicines frequently used in gynecology clinics on CYP3A4 and calculated the related titer of one-time dosage of Kampo medicine to GFJ in order to predict its effect on clinics.

\section{Material and Methods}

2.1. Crude Drugs and Kampo Formulation. Tokishakuyakusan (Dangguishaoyaosan) (daily dose for human) consists of $4.0 \mathrm{~g}$ of the root of Paeonia lactiflora (Peony Root), $4.0 \mathrm{~g}$ of the rhizome of Atractylodes lancea (Atractylodes Lancea Rhizome), $4.0 \mathrm{~g}$ of the tuber of Alisma orientale (Alisma Tuber), $4.0 \mathrm{~g}$ of the sclerotium of Wolfiporia cocos (Poria Sclerotium), $3.0 \mathrm{~g}$ of the rhizome of Cnidium officinale (Cnidium Rhizome), and $3.0 \mathrm{~g}$ of the root of Angelica acutiloba (Japanese Angelica Root). Kamishoyosan (Jiaweixiaoyaosan) (daily dose for human) consists of $3.0 \mathrm{~g}$ of the root of Bupleurum falcatum (Bupleurum Root), $3.0 \mathrm{~g}$ of Paeony root, 3.0 of Atractylodes Lancea Rhizome, $3.0 \mathrm{~g}$ of Japanese Angelica Root, $3.0 \mathrm{~g}$ of Poria Sclerotium, $2.0 \mathrm{~g}$ of the fruit of Gardenia jasminoides (Gardenia Fruit), $2.0 \mathrm{~g}$ of the root bark of Paeonia suffruticosa (Moutan Bark), $1.5 \mathrm{~g}$ of the root and stolon, with (unpeeled) or without (peeled) the periderm, of Glycyrrhiza uralensis (Glycyrrhiza), $1.0 \mathrm{~g}$ of the rhizome, with (unpeeled) or without (peeled) the periderm, of Zingiber officinale (Ginger), and $1.0 \mathrm{~g}$ of the terrestrial part of Mentha arvensis var. piperascens (Mentha Herb). Keishibukuryogan (Guizhifulingwan) (daily dose for human) consists of $3.0 \mathrm{~g}$ of the bark of the trunk of Cinnamomum cassia (Cinnamon Bark), $3.0 \mathrm{~g}$ of Paeony root, $3.0 \mathrm{~g}$ of the seed of Prunus persica (Peach Kernel), $3.0 \mathrm{~g}$ of Poria Sclerotium, and $3.0 \mathrm{~g}$ of Moutan Bark. These crude drugs were purchased from Daikoshoyaku (Nagoya, Japan) and were standardized by Japanese Pharmacopoeia 17th Edition [17]. The quality managers of the distributer identified and certificated the plant species, and the voucher specimens are deposited in Department of Pharmacognosy, Graduate School of Pharmaceutical Sciences, Nagoya City University, Nagoya, Japan. The mixtures of the above crude drugs' formula or each $10 \mathrm{~g}$ of single crude drug were boiled in 20 times the weight of water for $30 \mathrm{~min}$ and filtered. The decoction was lyophilized to yield powdered extract. Lot number and the extract ratio yielded are shown in Table 1. These extracts were suspended in distilled water to prepare the stock solution at a concentration of $0.1 \mathrm{~g} / \mathrm{ml}$ and kept in $-20^{\circ} \mathrm{C}$ until use.

Original dried extracts of tokishakuyakusan (TJ-23, Lot\# 2150023010), kamishoyosan (TJ-24, Lot\# 2160024010), and keishibukuryogan (TJ-25, Lot\# 2150025010), that were also standardized by Japanese Pharmacopoeia 17th Edition [17], without any excipient as ethical Kampo extract formulation, were produced by and supplied from Tsumura Co., Ltd. (Tokyo).

Fingerprint patterns of these formulas were shown in Supplementary Figures 1-6. Each extract (25 mg) was suspended with $\mathrm{MeOH}(1 \mathrm{ml})$ and sonicated for $30 \mathrm{~min}$. The supernatant $(25 \mu \mathrm{l})$ was injected to HPLC with the following conditions: system, Shimadzu LC-10A $\mathrm{A}_{V P}$ (Kyoto, Japan); column, TSK-GEL ODS- $80_{\mathrm{TS}}(4.6 \times 250 \mathrm{~mm}$, Tosoh, Tokyo); mobile phase, $0.05 \mathrm{M} \mathrm{AcOH}-\mathrm{AcONH}_{4}$ buffer $(\mathrm{pH}$ 3.6) $/ \mathrm{CH}_{3} \mathrm{CN}$ 90:10 (0 min) - 0:100 (60 min), linear gradient; flow rate, $1.0 \mathrm{ml} / \mathrm{min}$; column temperature, $40^{\circ} \mathrm{C}$; and detection, $200-400 \mathrm{~nm}$ by a photodiode array detector. Some peaks were identified by the retention times and UV spectra of the standard compounds.

2.2. Materials. Pooled human liver microsomes, mixed gender (Lot\# 610016), were bought from Sekisui XenoTech, LLC (Kansas City, KS, USA). Testosterone and $n$-butyl $p$ hydroxybenzoate $(\mathrm{BHB})$ were bought from Nacalai Tesque (Kyoto, Japan). 6,7'-Dihydroxybergamottin (DHBG), $\beta$ $\mathrm{NADPH}$, and $6 \beta$-hydroxytestosterone were purchased from ChemFaces (Wuhan, Hubei, China), Oriental Yeast (Tokyo, Japan), and Sigma Aldrich (St. Louis, MO, USA), respectively. The purities of DHBG and $6 \beta$-hydroxytestosterone were more than $98 \%$ according to the attached sheets.

2.3. Grapefruit Juice (GFJ). A commercial GFJ (Lot\#, 18.5.20 CK/3B, Toropicana 100, KIRIN Tropicana Inc., Tokyo, Japan) was purchased at a supermarket in Nagoya, Japan, in May, 2018. Ten $\mathrm{ml}$ of this GFJ was diluted with $\mathrm{H}_{2} \mathrm{O}$ and lyophilized to yield $1.0 \mathrm{~g}$ of dried powder. This powder was suspended in distilled water to prepare the stock solution at a concentration of $0.1 \mathrm{~g} / \mathrm{ml}$ and kept in $-20^{\circ} \mathrm{C}$ until use. Five-times diluted GFJ with $\mathrm{MeOH}$ was filtrated through $0.45 \mu \mathrm{m}$ filter, and then the content of DHBG was measured using HPLC as follows: system, Shimadzu LC-10A $\mathrm{A}_{V P}$; column, Inertsil ODS$2(4.6 \times 250 \mathrm{~mm}$, GL Science, Tokyo, Japan); mobile phase, A, $0.5 \%$ formic acid; B, acetonitrile containing $0.5 \%$ formic acid; A/B 20:80 (0 min), 50:50 (2 min), and 50:50 (15 min); flow rate, $1.0 \mathrm{ml} / \mathrm{min}$; column temperature, $40^{\circ} \mathrm{C}$; detection, absorbance at $310 \mathrm{~nm}$; injection volume, $10 \mu \mathrm{l}$; retention time of DHBG, $14.1 \mathrm{~min}$. Linear regression of the concentration range of $0.2-2 \mu \mathrm{g} / \mathrm{ml}$ of DHBG in methanol by the peak-area was calibrated with the least-squares method $\left(r^{2}=0.999\right)$, and the concentration of DHBG in the sample was calculated by this regression formula.

2.4. Assay for CYP3A4 Inhibition. We used slightly modified methods using rat liver microsome fraction as crude enzymes reported previously [9]. In brief, $10 \mu \mathrm{l}$ of the sample solution, $75 \mu \mathrm{l}$ of $0.15 \mathrm{M}$ phosphate buffer ( $\mathrm{pH} 7.4$ ) containing 0.13 M EDTA, $1.0 \mu \mathrm{l}$ of $20 \mathrm{mM}$ testosterone (dissolved in $20 \%$ $\mathrm{MeOH})$, and $4 \mu \mathrm{l}$ of human liver microsome $(3 \mathrm{mg} / \mathrm{ml}$ diluted with phosphate buffer) were mixed and incubated at $37^{\circ} \mathrm{C}$ for $5 \mathrm{~min}$. Then, $10 \mu \mathrm{l}$ of $1 \mathrm{mM} \beta$-NADPH and $10 \mu \mathrm{l}$ of 6 $\mathrm{mM} \mathrm{MgCl} 2$ were added, mixed, and incubated at $37^{\circ} \mathrm{C}$ for $15 \mathrm{~min}$. The reaction was stopped by the addition of $100 \mu \mathrm{l}$ of 2-propanol containing $0.1 \mathrm{M} \mathrm{BHB}$ as an internal standard 


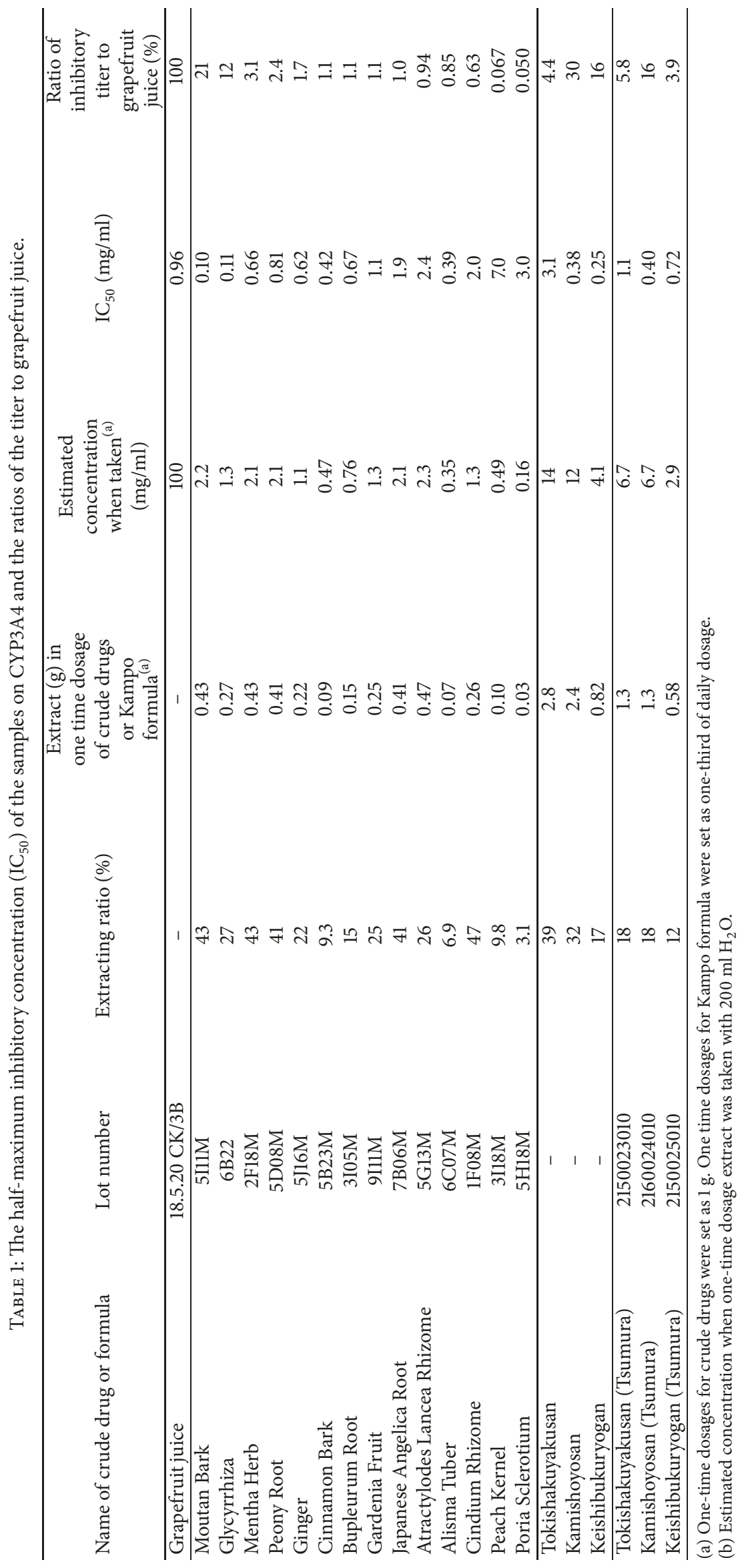




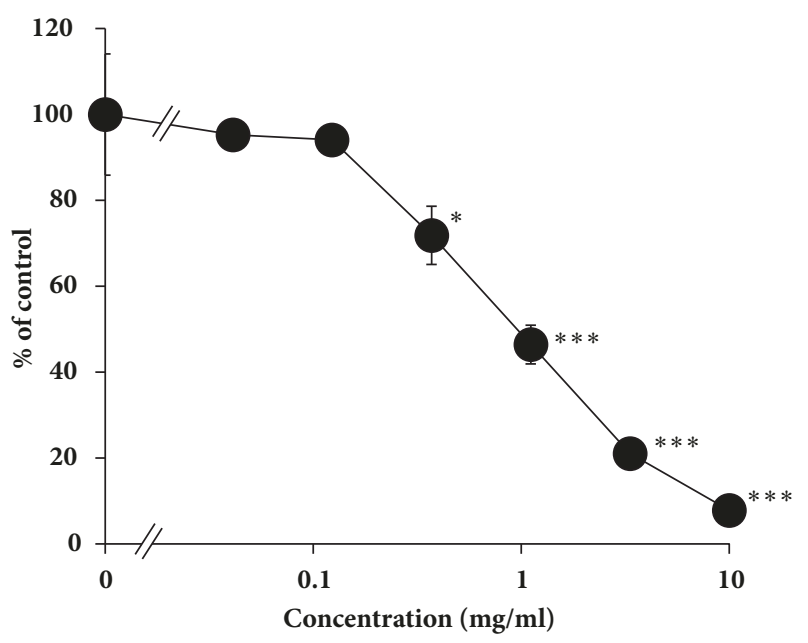

FIGURE 1: Inhibitory effect of the grapefruit juice (GFJ) used in this study on CYP3A4. Data are expressed as the mean $\pm \mathrm{SD}(n=3)$.

into the reactant and mixed vigorously. After centrifugation $(12,000 \times g, 5 \mathrm{~min}), 25 \mu \mathrm{l}$ of the supernatant was applied to the following HPLC analysis using the same system except for mobile phase $\mathrm{H}_{2} \mathrm{O} / \mathrm{MeOH}$ 40:60 and detection absorbance at $238 \mathrm{~nm}$. Retention times of testosterone and $\mathrm{BHB}$ were $23.0 \mathrm{~min}, 7.9 \mathrm{~min}$, and $\mathrm{BHB}$, respectively. The linear regression of the concentration range of $0.013-0.20$ $\mathrm{mM}$ of $6 \beta$-hydroxytestosterone by the peak-area ratio of $6 \beta$-hydroxytestosterone to $\mathrm{BHB}$ was calibrated with the least-squares method $\left(r^{2}=0.999\right)$. Data were expressed as relative activity (\%) that was the ratio of the content of $6 \beta$ testosterone incubated with the sample to that incubated without the sample. The assay was conducted in triplicate, and the mean \pm SD was exhibited. The half-maximum inhibitory concentration $\left(\mathrm{IC}_{50}\right)$ was calculated from the least square regression line made from 3 points that crossed $50 \%$ of the control logarithmic concentration values.

2.5. Statistics. Statistical analysis was carried out by one-way analysis of variance and Dunnett's multiple comparison $t$ test using PASW Statistics software (version 18, SPSS; IBM, Armonk, NY, USA). A probability value of less than 0.05 was considered statistically significant.

\section{Results}

3.1. Inhibitory Effect of Grapefruit Juice on CYP3A4. The grapefruit juice used in the present study contained $3.1 \mu \mathrm{g} / \mathrm{ml}$. This grapefruit juice inhibited CYP3A4 in dose-dependent manners with $\mathrm{IC}_{50}$ value of $0.96 \mathrm{mg} / \mathrm{ml}$ (Figure 1). This value is related to 1:104 diluted solution of the original grapefruit juice.

3.2. Inhibitory Effects of Tokishakuyakusan, Kamishoyosan, and Keishibukuryogan Extracts on CYP3A4. The extracts of tokishakuyakusan, kamishoyosan, and keishibukuryogan prepared in our laboratory or supplied by pharmaceutical industry inhibited CYP3A4 in dose-dependent manners (Figure 2). $\mathrm{IC}_{50}$ values of the extracts were shown in Table 1.

3.3. Inhibitory Effects of the Extracts of Crude Drugs Composing of Tokishakuyakusan, Kamishoyosan, and Keishibukuryogan on CYP3A4. The extracts of crude drugs composing of tokishakuyakusan, kamishoyosan, and keishibukuryogan prepared in our laboratory inhibited CYP3A4 in dosedependent manners (Figure 3 ). $\mathrm{IC}_{50}$ values of the extracts were shown in Table 1.

\section{Discussion}

In the present study, the extract of tokishakuyakusan, kamishoyosan, and keishibukuryogan, frequently prescribed Kampo formula in gynecological clinics, inhibited CYP3A4 in vitro, with $\mathrm{IC}_{50}$ value of $3.1,0.38$, and $0.25 \mathrm{mg} / \mathrm{ml}$. The one-time dosage of the extracts of tokishakuyakusan, kamishoyosan, and keishibukuryogan (3 times a day, t.i.d.) is 2.8, 2.4, and $0.82 \mathrm{~g}$, and when patients take the extract with a cup of water $(200 \mathrm{ml})$, the concentrations of the extracts are calculated as 14,12 , and $4.1 \mathrm{mg} / \mathrm{ml}$, respectively. These concentrations are $4.5,32$, and 16-times higher than their $\mathrm{IC}_{50}$ values, and these ratios seem large to cause the inhibition in vivo. However, since the results of CYP inhibitions in in vitro experiments tended to exhibit excessive high potency [14], in vitro results should be treated carefully.

In this study, the original concentration of GFJ used was $100 \mathrm{mg} / \mathrm{ml}$, and its IC $_{50}$ value on CYP3A4 was $0.96 \mathrm{mg} / \mathrm{ml}$. Since GFJ is the only natural substance to inhibit CYP3A4 in clinical studies [14], these values can be used as references to discuss the titer of inhibitory effect on CYP3A4 in clinics in comparison with in vitro inhibitory effects. The ratio of $\mathrm{IC}_{50}$ of GFJ to the concentration of original GFJ is calculated as 1:104; this value can be used as the coefficient for the extrapolation from in vitro results in the clinical situations. Ratio of inhibitory titer of the sample to GFJ is defined as the following formula:

$$
\begin{aligned}
& \text { Ratio of inhibitory titer to GFJ } \\
& \qquad=\frac{\mathrm{IC}_{50} \text { of } \mathrm{GFJ}}{\text { Conc. of GFJ }} \times \frac{\mathrm{IC}_{50} \text { of the target }}{\text { Conc. of the target }}
\end{aligned}
$$

Using this formula, the inhibitory effect of tokishakuyakusan, kamishoyosan, and keishibukuryogan extracts on CYP3A4 in clinics can be calculated as about $4.4 \%, 30 \%$, and $16 \%$ to that of GFJ (Table 1). The ratio 30\% might be possible in clinics when a patient takes about 3-fold amount of the prescription, and it is possible that excessive dosage of kamishoyosan might cause drug interaction with the substrate of CYP3A4 in clinics. On the other hand, since tokishakuyakusan extract needs 23-fold amount of the prescription to exhibit the inhibitory effect as same as GFJ, it is considered that the possibility of causing drug interaction in clinics might be low.

When we prepare decoction of Kampo formula, the variation of crude drugs from batch to batch must not be avoidable. In this study, we also evaluated the effect of ethical Kampo extract prescription of the same formula manufactured from 


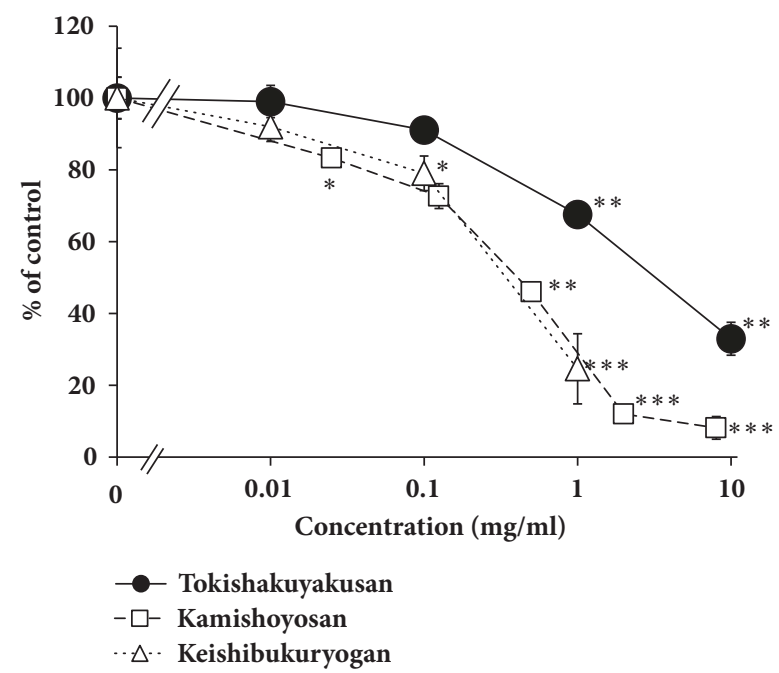

(a)

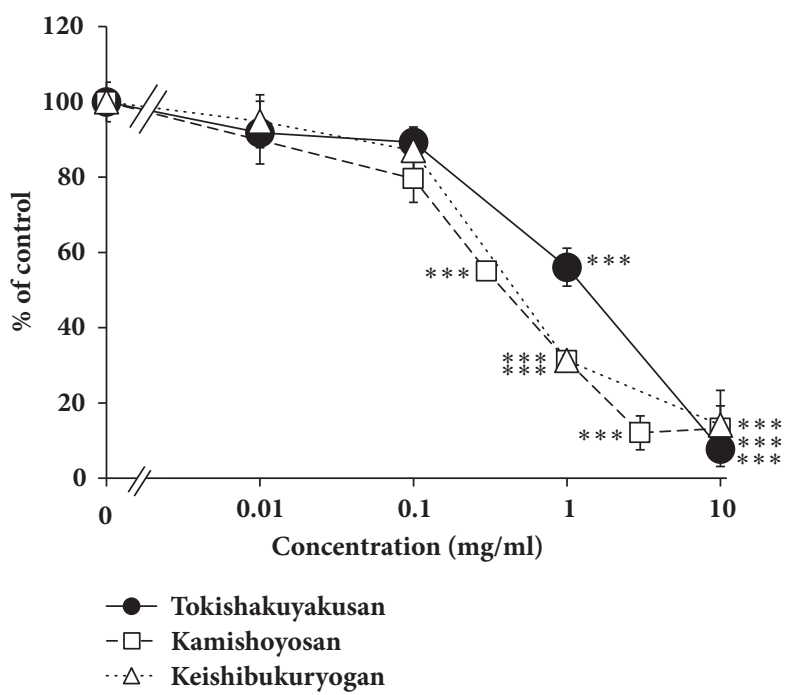

(b)

FIGURE 2: Inhibitory effect of tokishakuyakusan, kamishoyosan, and keishibukuryogan extracts on CYP3A4. (a) Each extract of Kampo formula was prepared from crude drug mixture described in Materials and Methods. (b) Each extract of Kampo formula was original dried extract of ethical Kampo extract formulation supplied from Tsumura Co., Ltd. Data are expressed as the mean \pm SD $(n=3)$.

pharmaceutical industry. Although the compositions and dosages of the formulas were the same, the extracting ratios of the products made in our laboratory were higher than those manufactured in the industry. This difference would be dependent on the scale of preparation; that is, the industry produced large scale of the extract at the same time though we prepared the extract of 1-day dosage in our laboratory. By this reason, $\mathrm{IC}_{50}$ value of keishibukuryogan extract prepared in the laboratory had lower than that of the extract supplied from the industry. However, although the extracting ratios of tokishakuyakusan and kamishoyosan prepared in the laboratory were larger than those supplied from the industry, $\mathrm{IC}_{50}$ values of the extract prepared in the laboratory were almost the same for kamishoyosan and larger for tokishakuyakusan compared with the extract from the industry. This may be caused by the differences of original crude drug batches used, and in order to provide drug information about Kampo medicine to clinics, we have to consider the variation of the products. Considering the inhibitory titers of both extracts, it is possible that excessive dosage of kamishoyosan may cause drug interaction with the substrate of CYP3A4 in clinics, but the possibilities of tokishakuyakusan and keishibukuryogan to cause drug interaction can be quite low. Indeed, in clinical study, when healthy females took ethical keishibukuryogan extract formulation for 7 days, it had no effect on CYP3A4 activity [18].

Among the herbal components of these three Kampo formulas, Moutan Bark and Glycyrrhiza had relatively high inhibitory effects on CYP3A4 in vitro. Among 50 crude drugs used in traditional Chinese medicine, Moutan Bark extract exhibited the highest inhibitory effect on CYP3A4 in vitro [19]. The inhibitory titer of Moutan Bark extract on CYP3A4 in clinics can be calculated as $21 \%$ to that of GFJ; it is possible that Moutan Bark may cause drug interaction with the substrate of CYP3A4 in clinics when the patients take excessive dosage. There are several reports that Glycyrrhiza (licorice) extract or its main ingredient glycyrrhizin inhibits CYP3A4 in vitro; its inhibitory titer was weak $[20,21]$. This study exhibited that the inhibitory titer of Glycyrrhiza extract was calculated as $12 \%$ to that of GFJ, and the possibilities of Glycyrrhiza to cause drug interaction with CYP3A4 substrate can be low.

In conclusion, this study provides the inhibitory titers of 3 Kampo formulas frequently used in gynecological clinics on CYP3A4 as quantitative forms and exhibited that these Kampo formulas would not cause drug interaction via CYP3A4 when the patients take regular dosage. Since it might be possible that kamishoyosan cause drug interaction via CYP3A4 in excessive dosage, clinical study of kamishoyosan might be demanded for the safe usage of Kampo medicines to prevent drug interaction.

\section{Data Availability}

The data used to support the findings of this study are available from the corresponding author upon request.

\section{Conflicts of Interest}

The authors of this manuscript have the following conflicts of interest: Takashi Matsumoto and Junko Watanabe are employees of Tsumura \& Co.

\section{Supplementary Materials}

Fingerprint patterns of the experimental materials prepared from crude drugs used in this study were shown in Supplementary Figures 1-6. (Supplementary Materials) 

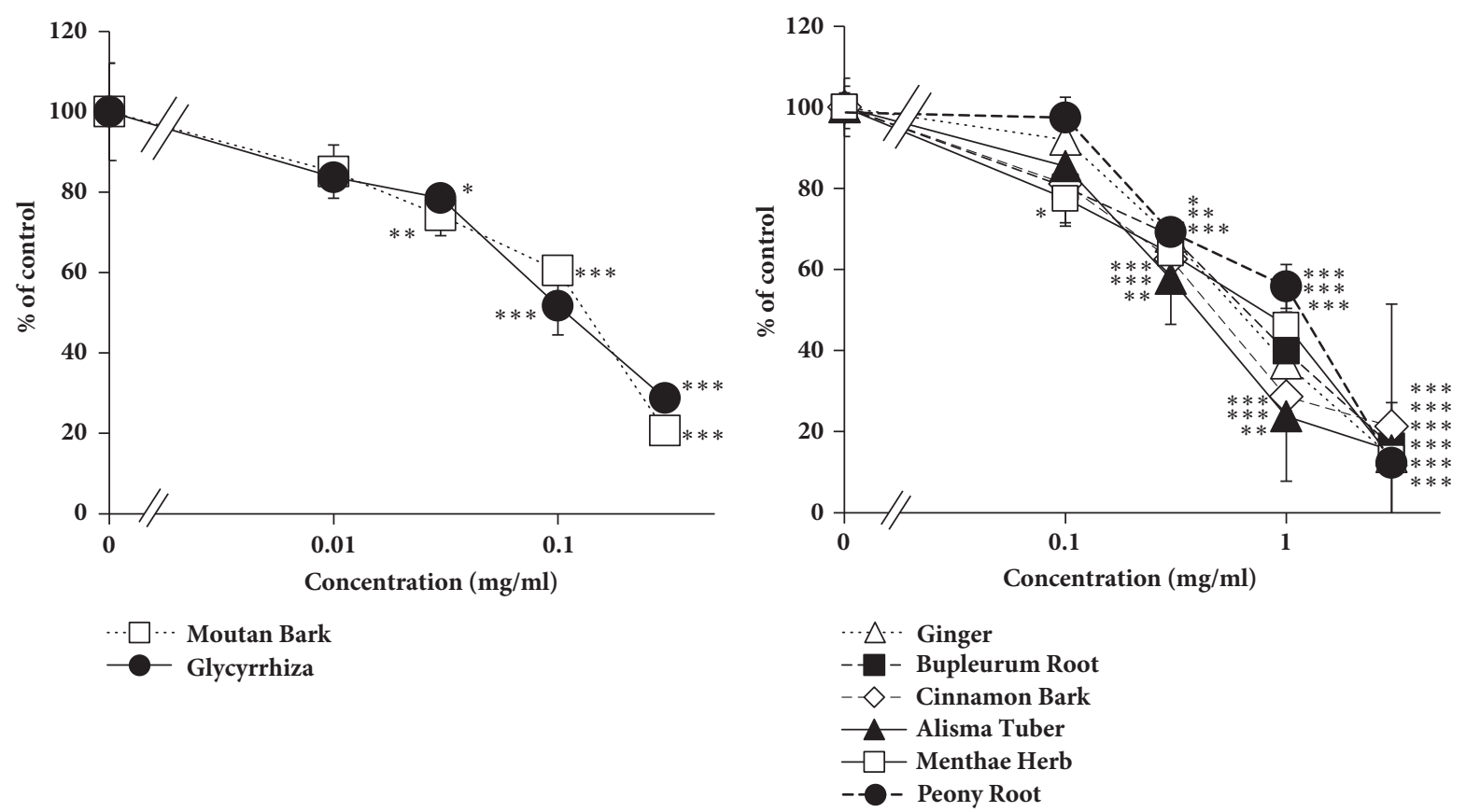

(a)

(b)

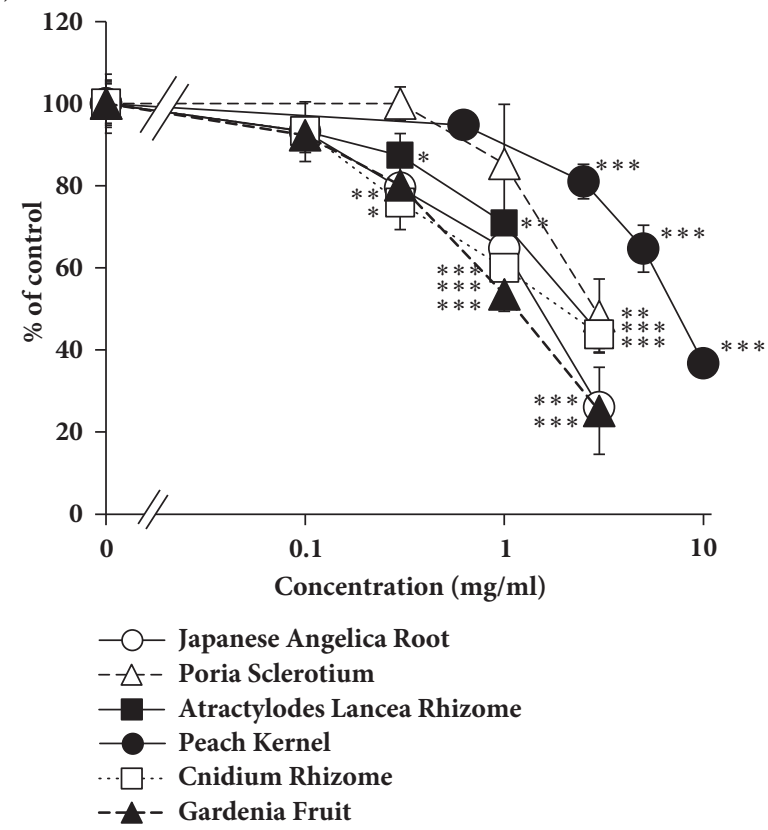

(c)

FIGURE 3: Inhibitory effect of the extracts of crude drugs composing tokishakuyakusan, kamishoyosan, and keishibukuryogan extracts on CYP3A4. Data are expressed as the mean $\pm \operatorname{SD}(n=3)$.

\section{References}

[1] A. Fugh-Berman and E. Ernst, "Herb-drug interactions: Review and assessment of report reliability," British Journal of Clinical Pharmacology, vol. 52, no. 5, pp. 587-595, 2001.

[2] E. Mills, P. Wu, B. C. Johnston, K. Gallicano, M. Clarke, and G. Guyatt, "Natural health product-drug interactions: A systematic review of clinical trials," Therapeutic Drug Monitoring, vol. 27, no. 5, pp. 549-557, 2005.
[3] M. L. Chavez, M. A. Jordan, and P. I. Chavez, "Evidence-based drug-herbal interactions," Life Sciences, vol. 78, no. 18, pp. 21462157, 2006.

[4] Q. Meng and K. Liu, "Pharmacokinetic interactions between herbal medicines and prescribed drugs: Focus on drug metabolic enzymes and transporters," Current Drug Metabolism, vol. 15, no. 8, pp. 791-807, 2014.

[5] D. Bailey, J. Spence, C. Munoz, and J. M. O. Arnold, "Interaction of citrus juices with felodipine and nifedipine," The Lancet, vol. 337, no. 8736, pp. 268-269, 1991. 
[6] M. Pirmohamed, "Drug-grapefruit juice interactions: two mechanisms are clear but individual responses vary," British Medical Journal, vol. 346, article f1, 2013.

[7] K. Iwanaga, K. Arimune, M. Miyazaki et al., "Effects of furanocoumarins in kampo extract-based medicines on rat intestinal absorption of CYP3A and P-glycoprotein substrate drugs in vivo," Archives of Pharmacal Research, vol. 35, no. 6, pp. 10551064, 2012.

[8] K. Iwanaga, M. Hayashi, Y. Hamahata et al., "Furanocoumarin derivatives in Kampo extract medicines inhibit cytochrome P450 3A4 and P-glycoprotein," Drug Metabolism and Disposition, vol. 38, no. 8, pp. 1286-1294, 2010.

[9] T. Makino, F. Mizuno, and H. Mizukami, "Does a kampo medicine containing schisandra fruit affect pharmacokinetics of nifedipine like grapefruit juice?” Biological \& Pharmaceutical Bulletin, vol. 29, no. 10, pp. 2065-2069, 2006.

[10] Natsum Kinoshita I, Yuriko Yamaguchi, Xiao-Long Hou, Kyoko Takahashi, and Koich Takahashi I, "Experimental adjustment on drug interactions through intestinal CYP3A activity in rat: impacts of kampo medicines repeat administered," EvidenceBased Complementary and Alternative Medicine, vol. 2011, Article ID 827435, 10 pages, 2011.

[11] K. Nobutani, J. Miyoshi, M. W. Musch et al., "Daikenchuto (TU100) alters murine hepatic and intestinal drug metabolizing enzymes in an in vivo dietary model: effects of gender and withdrawal," Pharmacology Research and Perspectives, vol. 5, no. 5, Article ID e00361, 2017.

[12] N. Okada, A. Murakami, S. Urushizaki, M. Matsuda, K. Kawazoe, and K. Ishizawa, "Extracts of immature orange (Aurantii Fructus Immaturus) and citrus unshiu peel (Citri Unshiu Pericarpium) induce P-glycoprotein and cytochrome P450 3A4 expression via upregulation of pregnane $\mathrm{X}$ receptor," Frontiers in Pharmacology, vol. 8, article 84, 2017.

[13] K. Ito, T. Satoh, Y. Watanabe et al., "Effects of Kampo Medicines on CYP and P-gp Activity in Vitro," Biological \& Pharmaceutical Bulletin, vol. 31, no. 5, pp. 893-896, 2008.

[14] M. J. Hanley, P. Cancalon, W. W. Widmer, and D. J. Greenblatt, "The effect of grapefruit juice on drug disposition," Expert Opinion on Drug Metabolism \& Toxicology, vol. 7, no. 3, pp. 267286, 2011.

[15] Japan Kampo Medicines Manufactures Association under the super vision of National Institute of Health Sciences, the affiliated institutions of the Ministry of Health, Labour and Welfare of Japan. Japan Kampo Medicines Manufactures Association under the super vision of National Institute of Health Sciences, Labour and Welfare of Japan, Handbook on OTC medicinal product in Kampo. Jiho, 2013.

[16] M. Terauchi, "Menopausal symptoms and the Kampo medicine: tokishakuyakusan, kamishoyosan, and keishibukuryogan, Recent Prog Kampo Med," Recent Progress of Kampo Medicine in Obstetrics and Gynecology, no. 33, pp. 23-30, 2016.

[17] Pharmaceutical and Medical Device Regulatory Science Society of Japan, Japanese Pharmacopoeia Seventeenth Edition (JP $X V I I)$, Pharmaceutical and Medical Device Regulatory Science Society of Japan, Tokyo, Japan, 2016.

[18] J. Saruwatari, C. Takaishi, K. Yoshida et al., "A herbal-drug interaction study of keishi-bukuryo-gan, a traditional herbal preparation used for menopausal symptoms, in healthy female volunteers," Journal of Pharmacy and Pharmacology, vol. 64, no. 5, pp. 670-676, 2012.

[19] L.-H. Pao, O. Y.-P. Hu, H.-Y. Fan, C.-C. Lin, L.-C. Liu, and P.-W. Huang, "Herb-drug interaction of 50 Chinese herbal medicines on CYP3A4 activity in vitro and in vivo," American Journal of Chinese Medicine, vol. 40, no. 1, pp. 57-73, 2012.

[20] S. Pandit, S. Ponnusankar, A. Bandyopadhyay, S. Ota, and P. K. Mukherjee, "Exploring the possible metabolism mediated interaction of Glycyrrhiza glabra extract with CYP3A4 and CYP2D6," Phytotherapy Research, vol. 25, no. 10, pp. 1429-1434, 2011.

[21] G. Li, C. Simmler, L. Chen et al., "Cytochrome P450 inhibition by three licorice species and fourteen licorice constituents," European Journal of Pharmaceutical Sciences, vol. 109, pp. 182190, 2017. 


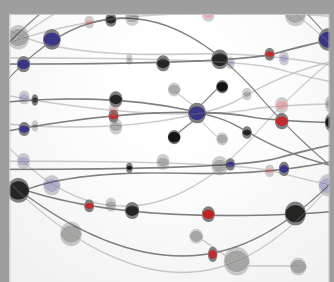

The Scientific World Journal
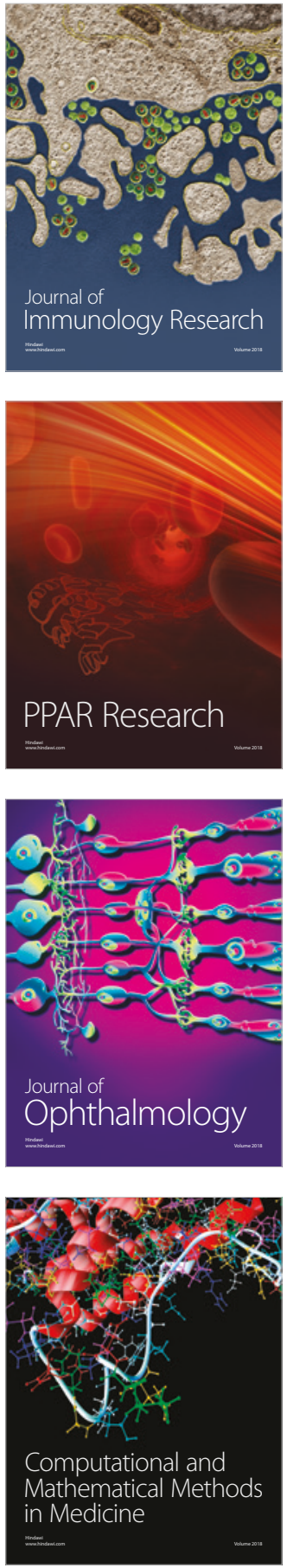

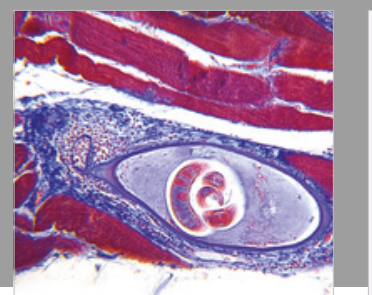

Gastroenterology Research and Practice

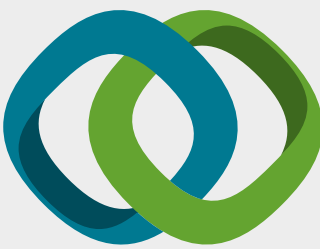

\section{Hindawi}

Submit your manuscripts at

www.hindawi.com
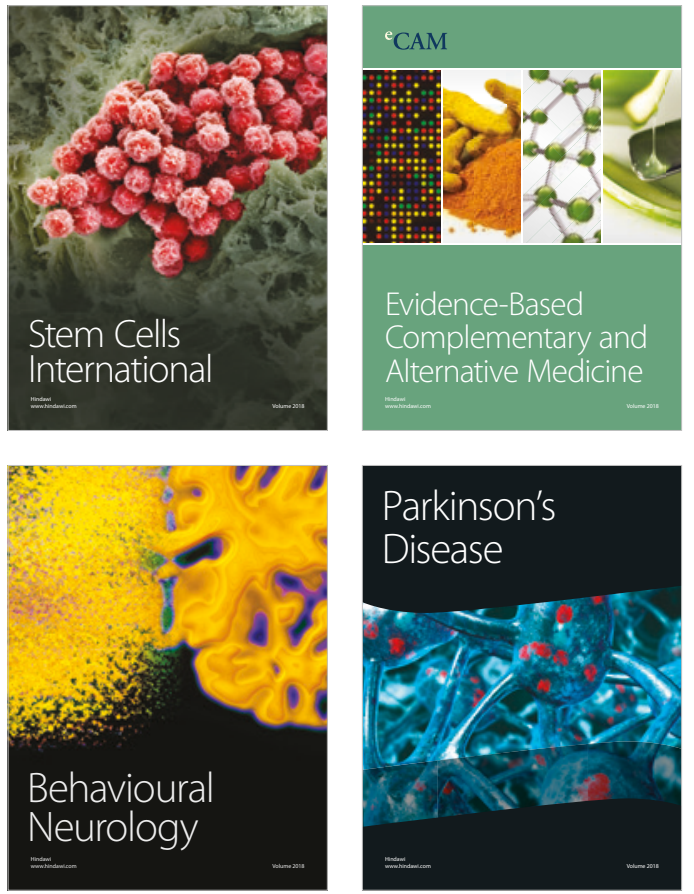

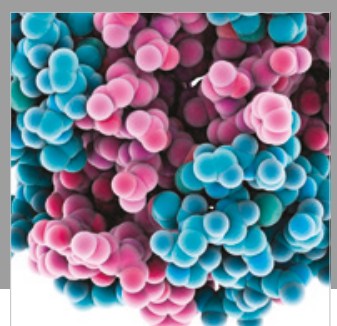

ournal of

Diabetes Research

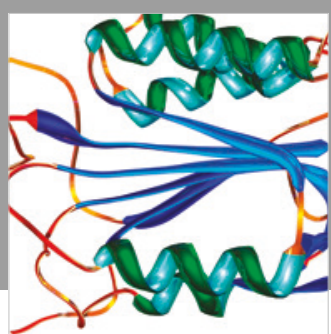

Disease Markers
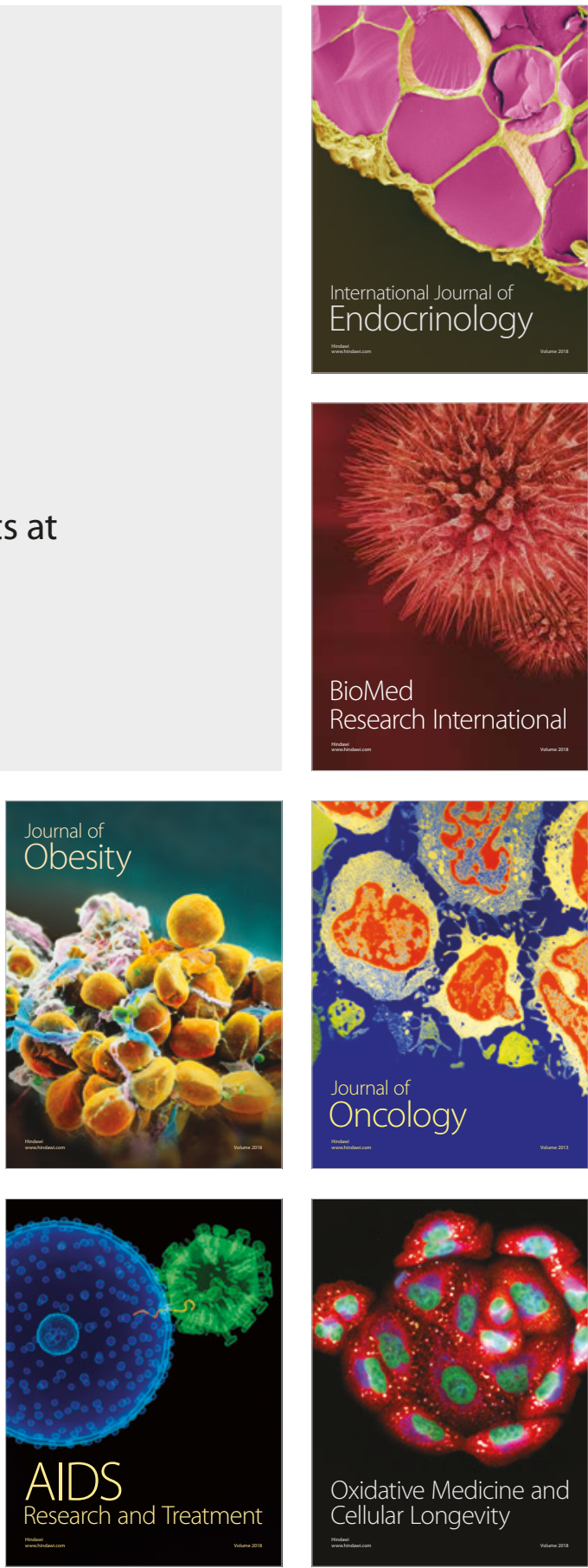